\title{
MICROMEGAS as a neutron beam profiler
}

\author{
I. Papadopoulos ${ }^{1}$, S. Andriamonje ${ }^{2}$, D. Cano-Ott ${ }^{4}$, A. Delbart ${ }^{1}$, J. Derré $^{1}$, S. Díez ${ }^{4}$, I. Giomataris ${ }^{1}$, \\ E.M. González-Romero ${ }^{4}$, F. Jeanneau ${ }^{1}$, D. Karamanis ${ }^{2}$, A. Leprêtre ${ }^{1}$, P. Pavlopoulos ${ }^{3}$, D. Villamarín ${ }^{4}$ \\ ${ }^{1}$ DSM/DAPNIA, CEA/Saclay, France, ${ }^{2}$ CEN Bordeaux-Gradignan, France \\ ${ }^{3}$ CERN, Geneva, Switzerland, ${ }^{4}$ CIEMAT, Madrid, Spain
}

\begin{abstract}
Two MICROMEGAS detectors, equipped with solid neutron converters, were tested on a neutron beam. The detectors have a $3 \mathrm{~mm}$ drift gap, a $100 \mu \mathrm{m}$ amplification gap and a strip pitch of $317.5 \mu \mathrm{m}$. The filling gas mixtures used for this test were argon/isobutane (90:10 and 98:2) and pure $\mathrm{CF}_{4}$. We present preliminary results of the operation of the detectors.

\section{INTRODUCTION}

Micromegas is a gaseous detector initially developed for tracking in high-rate, high-energy physics experiments [1]. Its properties open the way to use it in many other domains such as photodetection [2] and medical imaging. In this paper, the Micromegas performance in neutron detection applications is demonstrated.

A neutron time of flight facility (n_TOF) is being built at CERN [3], providing high neutron flux in the interval from $1 \mathrm{eV}$ to $250 \mathrm{MeV}$, to study neutron cross sections of almost any isotope, using targets of very modest mass, necessary for unstable or otherwise expensive materials. A precision of 1 $\mathrm{mm}$ on the measurement of the neutron beam shape at the experimental area (185 $\mathrm{m}$ downstream of the neutron source) is demanded. A Micromegas detector, equipped with a neutron conversion target is foreseen for this purpose.
\end{abstract}

Preliminary results with Micromegas detectors tested in a neutron beam at CENBG (Centre d'Etudes Nucléaires de Bordeaux - Gradignan) are presented.

\section{EXPERIMENTAL SETUP}

The Micromegas detector is a double-stage parallel plate chamber with a narrow amplification gap (Fig. 1). In the present tests, it consists of a $3 \mathrm{~mm}$ conversion gap and a $100 \mu \mathrm{m}$ amplification gap, separated by a metallic nickel micromesh (cathode), $3 \mu \mathrm{m}$ thick, with $37 \mu \mathrm{m}$ openings every $50 \mu \mathrm{m}$. Gold-plated copper strips (anode), $9 \mu \mathrm{m}$ thick, grounded through charge preamplifiers to define the anode potential, are printed using standard lithography on an epoxy plate, $1.6 \mathrm{~mm}$ thick. The strips, 384 in number, have a pitch of $317.5 \mu \mathrm{m}$. The third (drift) electrode is defined by an aluminum foil, $20 \mu \mathrm{m}$ thick, stretched on a G10 frame. The active surface of the detector is $12 \times 14 \mathrm{~cm}^{2}$.

Two Micromegas detectors have been tested, having different neutron converters, $500 \mathrm{~nm}$ thick, that were deposited by vacuum deposition on the inside face of the aluminum drift electrode and on one half of the surface. In the first one, the converter was a ${ }^{6} \mathrm{Li}$ layer, protected from oxidation by a very

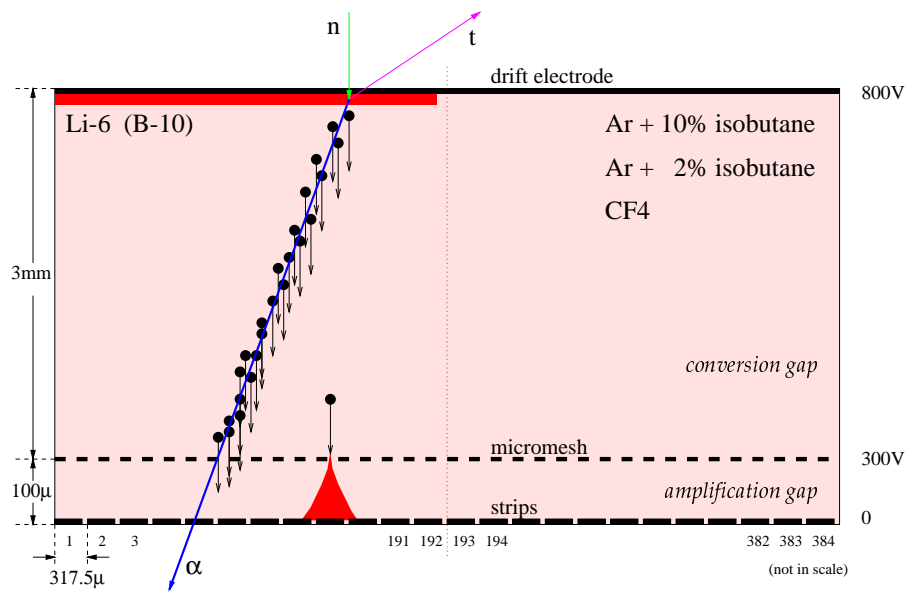

Figure 1: The Micromegas detector principle.

thin layer of aluminum, $25 \mathrm{~nm}$ thick, which was deposited over it. In the second one, the converter was a ${ }^{10} \mathrm{~B}$ layer.

The electronic cards used for the read-out of the detector strip signal are based on the GASSIPLEX chip. Each one consists of 96 multiplexed charge-sensitive-preamplifiers.

\section{DATA ANALYSIS}

The net strip signal is obtained from the raw signal, after the subtraction of the pedestal value and of an event by event baseline shift. This shift is due to the correlation of the pedestals for strips read by the same card. The net strip signal distribution is the superposition of the physical signals and a Gaussian centered at zero, with a standard deviation (sigma) reflecting the strip electronic noise, which was found to be of the order of 1000 electrons ENC. In order to discriminate the physical signal from the electronic noise, only strips with a net signal above 5 sigmas were accepted.

The strip clusters were adjusted by least squares fit to a linear function with a Gaussian shape at both extremes (rise and fall). The mean values of rise and fall edges provide the position of the end points of the track. The separation between these two values, FCS (fitted cluster size), is a reasonable estimation of the projected track length.

The neutron detection is based, on one hand, on the detection of the charged particles produced by neutron reactions in the target deposited on the drift electrode and, on the other hand, on the recoils coming from neutron elastic reactions in gas.

In the case of the ${ }^{6} \mathrm{Li}$ target, the outgoing particles of the reaction are an alpha and a triton, while in the case of the ${ }^{10} \mathrm{~B}$ target the outgoing particles of the reaction are an alpha 
and a ${ }^{7} \mathrm{Li}$. While the signals coming from those reactions are constraint in the target region of the detector (half of the active region), the signals coming from the elastic scattering of the neutrons in the gas are present in the whole volume of the detector.

To distinguish between the different particles, it is necessary to measure quantities characteristic to their nature, such as their energy deposition and their pathlength in the gas of the detector. Calculations show that for $600 \mathrm{keV}$ neutrons with the ${ }^{6} \mathrm{Li}$ target, particles depositing energy up to $85 \mathrm{keV}$ are protons, in the range from $325 \mathrm{up}$ to $505 \mathrm{keV}$ tritons and above $505 \mathrm{keV}$ mostly alphas. The proton region was not observed since the trigger threshold was always set above $85 \mathrm{keV}$. For the other two regions, it was not possible to use the energy deposition distribution alone, because of the saturation of the ADCs that distorted it. This saturation occurs in events of low strip multiplicity. Therefore, on the plot of the fitted cluster size (FCS) versus cluster charge (Fig. 2a), the saturated events are located at the lower part, well separated from the non-saturated ones. A clear separation between triton and alpha particles is observed. The charge cut that appears in this plot (at about 4500 ADC channels) is due to the trigger threshold on the mesh signal corresponding to $235 \mathrm{keV}$ of deposited energy.

Another way to identify the different charged particles is by using their $\mathrm{dE} / \mathrm{dx}$ in the gas. This makes necessary to know the true track length in the detector. With the Micromegas detector used for this test, only information for one spatial dimension can be obtained. Consequently, it is not possible to calculate the real pathlength of a particle and, therefore, its $\mathrm{dE} / \mathrm{dx}$. Nevertheless, for strip multiplicities high enough, the ratio of the cluster charge $(\mathrm{Q})$ over the FCS is a good approximation to the $\mathrm{dE} / \mathrm{dx}$ of the particle, otherwise it leads to an overestimation.

In a run with low gain (threshold at $500 \mathrm{keV}$ ), the proton recoils are not detected due to their limited energy deposition. For such a run, only tritons and alphas enter in the Q/FCS distribution, where the triton and alpha peaks are well separated (Fig. 2b). At higher gain (threshold at $235 \mathrm{keV}$ )), proton recoils are also detected. Their Q/FCS distribution overlaps with the triton and alpha peaks. Since in the non-target region only proton recoils are detected, it is possible to subtract the proton contribution to the $\mathrm{Q} / \mathrm{FCS}$ distribution of the events in the target region, to extract the triton and alpha contribution. In Fig. 2(c,d) are shown the proton (solid line), triton and alpha (dashed line) contributions to the Q/FCS distribution for two high gain runs: the triton and alpha peaks are always well separated and the proton contribution is in accordance with the hydrogen concentration in the gas mixture.

Two methods were used to estimate the spatial resolution. The first one is based on the starting point of the track and the second on the centroid of the cluster. The trajectories of charged particles produced in the target, projected on the strip plane, starting in the target region and ending in the non-target region provide an unambiguous starting point. As mentioned above, the strip cluster of each reconstructed event is fitted in
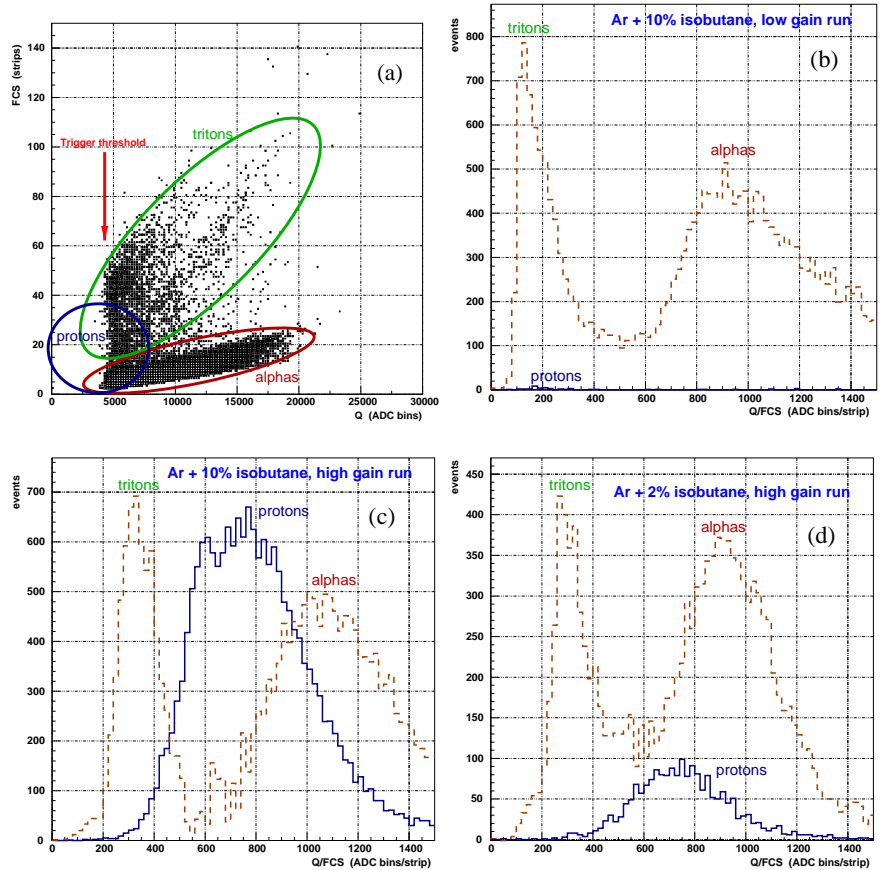

Figure 2: Fitted cluster size versus cluster charge (a) and Q/FCS distribution for proton recoils (solid line), tritons and alphas (dashed line) with (b) low gain, argon/isobutane (90:10), (c) high gain, argon/isobutane (90:10) and (d) high gain, argon/isobutane (98:2): In (b) no proton recoils are observed (cut by threshold), while from (c) to (d) the number of proton recoils drops according to the hydrogen concentration in the gas.

order to estimate the starting and ending position of the particle, $m_{1}$ and $m_{2}$ respectively $\left(m_{1}<m_{2}\right)$. In Fig. 3 is shown the distribution of $m_{2}$ versus $m_{1}$. The edge of the ${ }^{6} \mathrm{Li}$ target is clearly seen, due to the tritons that give high strip multiplicity signals. Selecting the events with an FCS higher than a certain value (optimized to 30 ) to enlarge the region where the starting point is unambiguous, the above mentioned function is fitted the $m_{1}$ distribution. The resulting spatial resolution is about $300 \mu \mathrm{m}$.
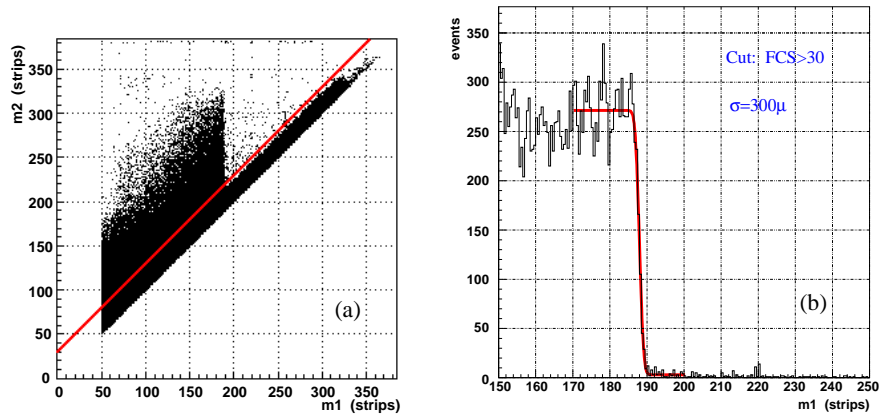

Figure 3: (a) $\mathrm{m} 2$ versus $\mathrm{m} 1$ (see text) and (b) $\mathrm{m} 1$ distribution after the cut FCS $>30$.

This method is based on the selection of events in a particular target region, allowing the use of high strip multiplicities. It cannot be used for a profiler, but it is useful for the estimation of a lower limit of the spatial resolution.

The second method, based on the centroid distribution 
of the events, can be applied to a profiler, since it does not require an event selection in a particular region of the detector. Lower the strip multiplicity is, closer the centroid value is to the neutron interaction position (track starting point). So, contrary to the first method, the events of high strip multiplicity degrade the spatial resolution. A cut on FCS $(\mathrm{FCS}<4.5)$ results in a rejection of $53 \%$ of the events and an improvement by a factor three on the spatial resolution. As expected, the spatial resolution is slightly degraded (about $400 \mu \mathrm{m}$ ). Nevertheless, this spatial resolution is well satisfying the $n_{-}$TOF prospect.

\section{Discussion}

Monte Carlo simulation shows that the deposited energy of the charged particles from the converter in the gas is higher than $80 \mathrm{keV}$, whatever the neutron energy is. Therefore, a threshold lower than $80 \mathrm{keV}$ is sufficient to reach their full detection. In this case, the neutron detection efficiency depends only on the cross section of the reaction of neutrons with the target.

Since the charged particle ionization is quite high, the conversion gap of the detector can be decreased (down to 1 $\mathrm{mm}$ ) to improve the spatial resolution, without affecting the detection efficiency. The drift time for a conversion gap of 3 $\mathrm{mm}$ is about $100 \mathrm{~ns}(25 \mathrm{~ns})$ for $\mathrm{Ar}\left(\mathrm{CF}_{4}\right)$. The reduction of the conversion gap down to $1 \mathrm{~mm}$ would also improvethe time resolution. Using fast electronics and a gap of $1 \mathrm{~mm}$, a time resolution of $1 \mathrm{~ns}$ can be obtained.

The proton recoil signals from the gas can be seen as a competitive process for the neutron detection, for energies above few $\mathrm{keV}$, for which the recoil rate becomes larger than the conversion rate. For lower energies, the neutron conversions dominate, as the cross section varies as $1 / \sqrt{\mathrm{E}_{n}}$. Therefore, a Micromegas detector with a converter and an argon/isobutane gas mixture can be used for the detection of neutrons in a wide energy range, from thermal to few $\mathrm{MeV}$ neutrons.

\section{CONCLUSions}

The performance of the Micromegas neutron profiler detector was studied in the energy range from $240 \mathrm{keV}$ up to $1 \mathrm{MeV}$. The results indicate an excellent background rejection with a high detection efficiency for the charge produced by the neutron capture in the converter. The conversion efficiency in the neutron converters tested was of the order of $10^{-4}$, which is reasonable for a beam profiler in a high flux neutron beam. A spatial resolution less than $400 \mu \mathrm{m}$ has been achieved, meeting the requirements for the n_TOF facility. The behavior was stable during the whole running period (10 days). It can provide safe operation and an accurate measurement of the relative neutron beam intensity and spatial profile.

\section{REFERENCES}

[1] Y. Giomataris, Ph. Rebourgeard, J.P. Robert and G. Charpak, NIM A376 (1996) 29.

[2] J. Derré et al., NIM A449 (2000) 314.

[3] "Proposal for a Neutron Time of Flight Facility", CERN/SPSC 99-8, SPSC/P310, 17 March 1999. 